fibromyalgia symptoms (AUC ${ }_{95 \%} \mathrm{cl}: 0.474[0.313-0.581], \mathrm{p}=0.409$ ), and metabolic syndrome score (AUC ${ }_{95 \% \text { C.I }}: 0.546[0.423-0.668], p=0.480$ )

Conclusion: Fibromyalgia women responded to the stress of pain by increasing the serum level of ACTH which effectively improves the clinical feature of fibromyalgia symptoms, but at the same time elevates the score of metabolic syndrome. Therefore, assessment of serum level of ACTH can serve as a predictor and discriminator of fibromyalgia comorbidity.

References:

[1] Acosta-Manzano P, Segura-Jiménez V, Estévez-López F, Álvarez-Gallardo IC, Soriano-Maldonado A, Borges-Cosic M, Gavilán-Carrera B, Delgado-Fernández M, Aparicio VA. Do women with fibromyalgia present higher cardiovascular disease risk profile than healthy women? The al-Ándalus project. Clin Exp Rheumatol. 2017; 35 Suppl 105(3):61-67.

[2] Marwan S.M. Al-Nimer, Talar A.M. Mohammad, Avin M.A. Maroof. Dysfunction of anterior pituitary gland in women patients with recent fibromyalgia: A cross-sectional observational study. Electron J Gen Med 2018;15(4):em58

[3] Soldatovic I, Vukovic R, Culafic D, Gajic M, Dimitrijevic-Sreckovic V. siMS score: simple method for quantifying metabolic syndrome. PLoS One. 2016; 11(1):e0146143

Disclosure of Interests: : None declared

DOI: 10.1136/annrheumdis-2020-eular.1087

\section{THU0466 EARLY DIAGNOSIS IS ASSOCIATED WITH LESS DISEASE SEVERITY AND BETTER OUTCOME IN FIBROMYALGIA SYNDROME: A TRICENTRIC PROSPECTIVE ANALYSIS OF A COHORT OF 370 PATIENTS}

A. Moshrif ${ }^{1}$, W. Gouda ${ }^{2}$, M. H. Abu-Zaid ${ }^{3} .{ }^{1}$ Al Azhar University, Assiut, Egypt; ${ }^{2}$ Aswan University, Aswan, Egypt; ${ }^{3}$ Tanta University, Tanta, Egypt

Background: Delayed diagnosis of fibromyalgia (FM) has been reported to be associated with more economic burden, healthcare utilization and worse response to treatment ${ }^{1,2}$. However, its impact on the patients' symptomatology and disease severity is still underestimated.

Objectives: to evaluate the effect of diagnostic delay (DD) on FM severity and disease assessment parameters.

Methods: in this cross sectional study, $370 \mathrm{FM}$ patients were prospectively interviewed. Information about DD, widespread pain index (WPI), symptom severity scale (SSS), total severity scale (SSS+WPI) and number of tender points were collected. We proposed to classify our patients into 3 categories; early diagnosis (ED $\leq 2$ years; 83 patients), late diagnosis (LD: >2-7 years; 198) and very late diagnosis (VLD >7 years; 89 patients).

Results: the mean age of patients was $33.9( \pm 9.8)$ and $79.4 \%$ were female. The mean for DD was $5.6( \pm 3.6)$ while the means for SSS, total scale and tender points were $7.8( \pm 1.6), 16.46( \pm 4.1), 14.31( \pm 2.3)$ respectively. A significant correlation has been found for DD with SSS $(r=0.14)$, total scale $(r=0.37)$ and tender points $(r=0.16)$ but not with WPI $(r=0.059)$.

Comparing the three categories, the mean for SSS was $7.54( \pm 1.6), 7.73( \pm 1.4)$ and $8.25( \pm 1.7)$ in the groups of ED, LD and VLD respectively $(P=0.008)$ while the mean for the total scale was $15( \pm 3.8), 15.95( \pm 3.8)$ and $18.96( \pm 4.4)$ respectively $(P=0.000)$ and the mean for tender points was $13.7( \pm 2.3), 14.35( \pm 2.1)$ and $14.77( \pm 2.8)$ respectively $(P=0.011)$. The mean for WPI did not significantly differ as it was $7.45( \pm 2.8), 7.8( \pm 3.6)$ and $7.18( \pm 4.8)$ in the groups of ED, LD and VLD respectively $(P=0.415)$.

Conclusion: early diagnosis of FM is associated with low SSS, total severity scale and tender points reflecting a better outcome and a less disease severity. References:

[1] Hughes G, Martinez C, Myon E, Taïeb C, Wessely S: The impact of a diagnosis of fibromyalgia on health care resource use by primary care patients in the UK: an observational study based on clinical practice.Arthritis Rheum 2006, 54(1):177-83.

[2] Choy et al., A patient survey of the impact of fibromyalgia and the journey to diagnosis BMC Health Services Research 2010, 10:102.

Disclosure of Interests: : None declared

DOI: 10.1136/annrheumdis-2020-eular.5504

\section{THU0467 CONCEPTS AND PERCEPTIONS ABOUT FIBROMYALGIA DIAGNOSIS, MONITORING AND TREATMENT AMONG COLOMBIAN RHEUMATOLOGISTS, PHYSIATRIST AND PAIN PHYSICIAN}

D. G. Fernández-Ávila1 ${ }^{1}$ D. Rincón-Riaño ${ }^{2}$, J. Gutiérrez ${ }^{1} .{ }^{1}$ Rheumatology Unit, Department of Internal Medicine, Hospital Universitario San Ignacio - School of Medicine, Pontificia Universidad Javeriana. Bogotá, Colombia, Bogota,
Colombia; ${ }^{2}$ Rheumatology Service, Hospital Militar Central - Universidad Militar Nueva Granada. Bogotá, Colombia, Bogota, Colombia

Background: Fibromyalgia is a chronic disease characterized by the presence of widespread and persistent musculoskeletal pain associated with a variety of symptoms. The concepts and perceptions around diagnosis and treatment of fibromyalgia among physicians are not objectively known. The purpose of this study is to obtain objective data through a survey and describe the concepts and perceptions about the diagnosis, treatment and treatment of fibromyalgia among colombian rheumatologists, physiatrist and pain physicians

Objectives: The main purpose of this study is to obtain objective data on this subject and describe the concepts and perceptions about the diagnosis, treatment and monitoring of FM among colombian rheumatologists, physiatrist and pain physicians.

Methods: Cross-sectional study. Through a focus group in which two rheumatologists and one expert in qualitative research methods participated, a survey was designed to evaluated the perceptions and concepts that rheumatologists, physiatrist and pain physicians have on the diagnosis and treatment of fibromyalgia. The survey was self-applied anonymously through the internet.

Results: Survey applied to 139 rheumatologists, 99 physiatrist and 81 pain physicians. 35 rheumatologists $(25.2 \%), 17$ physiatrist $(17.1 \%)$ and 58 pain physicians $(71.6 \%)$ consider that there is not enough evidence to recognize fibromyalgia as a disease. 45 rheumatologists (32.4\%), 86 physiatrist ( $86 \%$ ) and 73 pain physicians (90.1\%) consider that the 1990 ACR (American college of Rheumatology) criteria are not sufficient to diagnose fibromyalgia, despite the fact more than $90 \%$ of them use the criteria as a tool to approach the diagnosis when suspecting fibromyalgia. The most formulated medications for managing fibromyalgia are antidepressants and is used by more than $80 \%$ of the respondents, followed by antiepileptics in pain physician (88.9\%) but less than physiatrists and rheumatologists (66.6 \% and $64.7 \%$ respectively), and analgesic much more for pain physician and physiatry and less for rheumatologists (84 \%, $75.7 \%$ and $26.6 \%$ respectively). All respondents consider that the patient with fibromyalgia should have a multidisciplinary approach. Most doctors of the three specialties believe that physiatrist should be the leaders of interdisciplinary management in the treatment of fibromyalgia patients.

Conclusion: We present objective information on the perceptions of fibromyalgia among a group of Colombian rheumatologists, physiatrist and pain physician, documenting a frequent use of the ACR 1990 classification criteria. As regards treatment, a high percentage use of antidepressants and antiepileptic. Most believe that physiatrist should be the leaders of interdisciplinary management in the treatment of fibromyalgia patients.

\section{References:}

[1] Mu R, Li C, Zhu J-X, Zhang X-Y, Duan T-J, Feng M, et al. National survey of knowledge, attitude and practice of fibromyalgia among rheumatologists in China. Int J Rheum Dis. 2013;16:258-63.

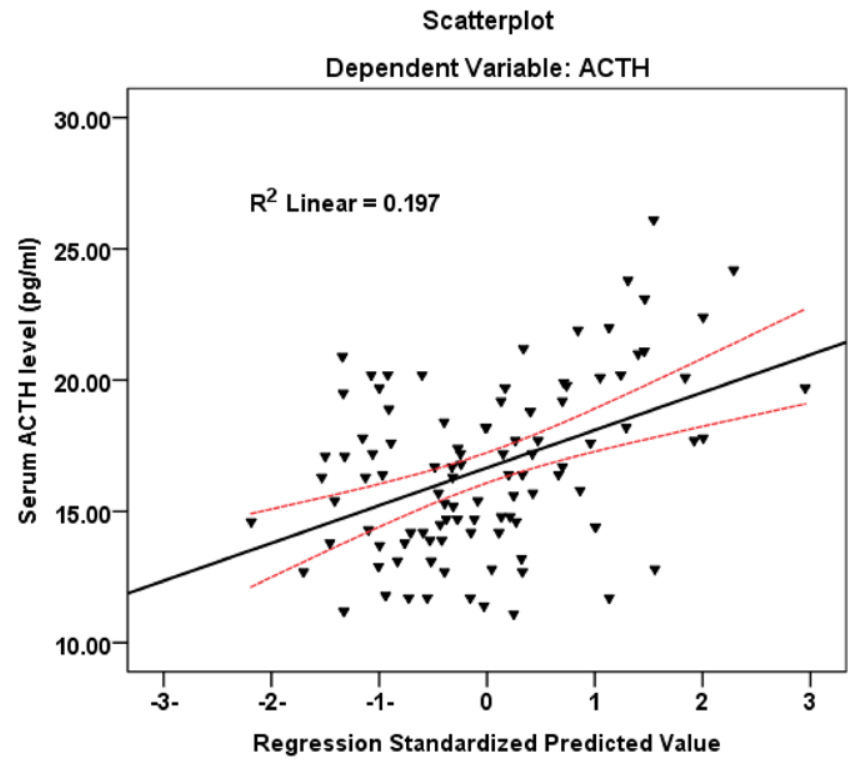

Figure 1. Multivariable regression analysis with posthoc ANOVA test showed significant correlations between serum level adrenocorticotrophic hormone as a dependent variable with the score $s$ of fibromyalgia impact questionnaire and metabolic syndrome, and the number of tender points. $R=0.443, F=7.777, P<0.001$, prediction $19.7 \%$ ), 
[2] Arshad A, Kong KO. Awareness and perceptions of fibromyalgia syndrome: a survey of Malaysian and Singaporean rheumatologists. Singapore Med J. 2007;48:25-30.

[3] Arshad A, Kong KO, Ooi KK. Awareness and perceptions of fibromyalgia syndrome: a survey of southeast asian rheumatologists. J Clin Rheumatol. 2007;13:59-62.

[4] Bloom S, Ablin JN, Lebel D, Rath E, Faran Y, Daphna-Tekoah S, et al. Awareness of diagnostic and clinical features of fibromyalgia among orthopedic surgeons. Rheumatol Int. 2013;33:927-31.

[5] Clark P, Paiva ES, Ginovker A, Salomón PA. A patient and physician survey of fibromyalgia across Latin America and Europe. BMC Musculoskelet Disord. 2013;14:188.

Disclosure of Interests: : None declared

DOI: 10.1136/annrheumdis-2020-eular.5213

\section{THU0468 THERAPIES IN RHEUMATIC AND MUSCULOSKELETAL DISEASES: AN OVERVIEW OF SYSTEMATIC REVIEWS INFORMING THE 2020 EULAR RECOMMENDATIONS FOR INTRA-ARTICULAR THERAPIES INCLUDING SYNOVIORTHESIS}

S. C. Rodriguez-García ${ }^{1}$, R. Castellanos-Moreira ${ }^{2}$, J. Uson Jaeger ${ }^{3}$, E. Naredo ${ }^{4}$, L. Carmona ${ }^{5}$ on behalf of the EULAR Intra-articular Therapies Task Force. ${ }^{1}$ Hospital de La Princesa, Madrid, Spain; ${ }^{2}$ Hospital Clínic de Barcelona, Barcelona, Spain; ${ }^{3}$ Hospital de Móstoles, Móstoles, Spain; ${ }^{4}$ Hospital Universitario Fundación Jiménez Díaz, Madrid, Spain; ${ }^{5}$ InMusc Instituto de Salud Musculoesquelética, Madrid, Spain

Background: Intra-articular therapy (IAT) is subject to wide variability and there are gaps in the evidence on its efficacy and safety.

Objectives: To assess the efficacy and safety of frequently used IATs to inform a EULAR Taskforce.

Methods: We performed an overview of systematic reviews (SR) of randomised clinical trials (RCT) assessing efficacy and safety of IAT in adults with RMDs. MEDLINE was searched until January 2019. SRs were assessed with the AMSTAR-2 tool. Critically low-confidence SRs were excluded.

Results: Of 159 articles identified, 42 were reviewed in detail and 15 met the inclusion criteria (146 RCTs). The populations included were mainly knee osteoarthritis (OA) in $10 \mathrm{SRs}$, rheumatoid arthritis (RA) in 3, hip and temporo-mandibular (TM) OA and shoulder adhesive capsulitis in $1 \mathrm{SR}$ each.

In knee OA, Hyaluronic Acid (HA) showed a modest benefit over placebo for pain and function but with unclear clinical significance in some studies. Platelet rich plasma (PRP) showed a small effect over HA only for function. Mesenchymal stem cells (MSC) performed better than PRP and HA for some outcomes, however, in studies with high risk of bias. Intra-articular Glucocorticoids (GC) were better than placebo (PBO) for pain and function.

More adverse events $(A E)$ were seen in the PRP group compared with $\mathrm{HA}$ and for $\mathrm{HA}$ compared with $\mathrm{PBO}$ including serious $\mathrm{AE}$ each in $1 \mathrm{SR}$ on knee OA. Results for other included diseases are shown in table 1.

Conclusion: Most of the SRs assessed had results of low confidence. HA and GC showed a small, short term benefit in knee arthritis in OA and RA compared to PBO. High risk of bias prevents conclusions on the efficacy of PRP and MSC in knee OA. More AE were reported in PRP and HA treated groups.

Disclosure of Interests: : Sebastian C Rodriguez-García Speakers bureau: Novartis Farmaceutica, S.A., Merck Sharp \& Dohme España, S.A., Sanofi Aventis, UCB Pharma, Raul Castellanos-Moreira Speakers bureau: Lilly, MSD, Sanofi, UCB, Jacqueline Uson Jaeger: None declared, Esperanza Naredo: None declared, Loreto Carmona Grant/research support from: Novartis Farmaceutica, SA, Pfizer, S.L.U., Merck Sharp \& Dohme España, S.A., Roche Farma, S.A, Sanofi Aventis, AbbVie Spain, S.L.U., and Laboratorios Gebro Pharma, SA (All trhough institution)

DOI: 10.1136/annrheumdis-2020-eular.645

\section{THU0469 \\ QUANTIFYING THE PLACEBO EFFECT AFTER INTRA- ARTICULAR INJECTIONS: IMPLICATIONS FOR TRIALS AND PRACTICE}

S. C. Rodriguez-García ${ }^{1}$, R. Castellanos-Moreira ${ }^{2}$, J. Uson Jaeger $^{3}$, E. Naredo ${ }^{4}$, L. Carmona ${ }^{5}{ }^{1}$ Hospital de La Princesa, Madrid, Spain; ${ }^{2}$ Hospital Clínic de Barcelona, Barcelona, Spain; ${ }^{3}$ Hospital Universitario de Móstoles, Móstoles, Spain; ${ }^{4}$ Hospital Universitario Fundación Jiménez Díaz, Madrid, Spain; ${ }^{5}$ InMusc Instituto de Salud Musculoesquelética, Madrid, Spain

Background: In recent years, diverse compounds for intra-articular administration were brought into the market with a subsequent significant and heterogeneous literature production. Understanding the efficacy of intra-articular therapies
(IAT) on pain implies bearing in mind the related placebo (PBO) effect. To date, most studies analyzing it were focused on the compound being administered rather than the route of administration.

Objectives: We aimed at evaluating the size of the PBO effect after intra-articular injections.

Methods: We conducted an overview of systematic reviews (SRs) including randomized-controlled trials (RCTs) of frequently used IAT. SRs with a saline solution PBO arm and high-confidence results according to the AMSTAR-2 tool were selected for analysis.

Data on the change in pain in the PBO arms from baseline to 3-6 and 12-16 weeks after the IA procedure was extracted. The standardized mean differences (SMD) from baseline were calculated as the ratio between the size of the intervention effect in each study and the variability observed in that study. A meta-analysis was then performed using an inverse-variance random-effects model in Review Manager 5.3. The overall effect sizes obtained refer to versions of the SMD, which corresponds to the Hedges' (adjusted) g. e.g. a "g" of 1 indicates the two groups being compared differ by 1 standard deviation and so on. Results: Two SR were included comprising 50 RCTs, 44 not meeting inclusion criteria were excluded so pain, measured by visual analogue scale (VAS) and Lequesne index, was retrieved from $6 \mathrm{RCT}$.

At 3-6 weeks, an SMD $[95 \% \mathrm{Cl}]=0.74[0.47-1.00]$ was found. One study showing too large an effect was excluded after conducting sensitivity analysis resulting in a significant reduction of heterogeneity with an SMD $=0.62$ [0.45-0.79] (Fig.1) At $12-16$ weeks, we found a SMD $=0.33$ [0.13-0.52] (Fig.2)

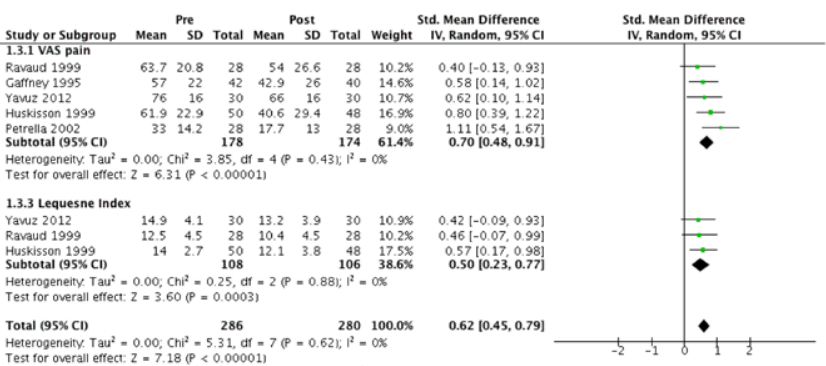

Figure 1. Forest plot for intra-articular PBO effect at 3-6 weeks after injection.

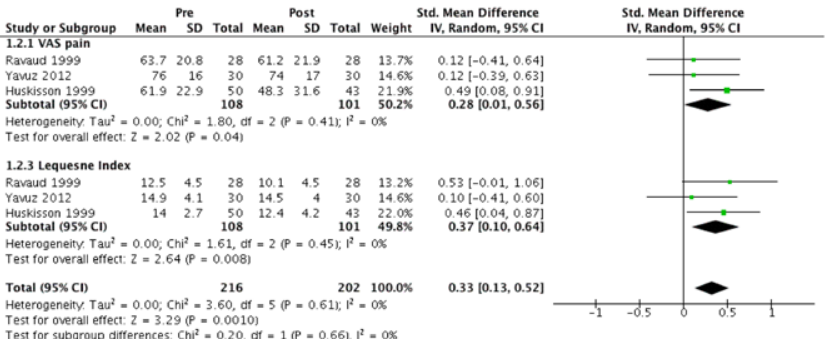

Figure 2. Forest plot for intra-articular PBO effect at 12-16 weeks after injection.

Using the interpretation suggested by Cohen ${ }^{1}$, our results would confirm a moderate to large effect of IA saline (PBO) at 3-6 weeks with a subsequent reduction to a small but persistent effect at 12-16 weeks.

Conclusion: Our results showed a moderate to large short-term effect of intra-articular PBO that persisted on the mid-term although reduced. Based on these findings we suggest this effect should be considered when assessing the efficacy of IAT in RCTs and also in clinical practice where it could be maximized as well.

\section{References:}

[1] Cohen J. Statistical Power Analysis in the Behavioural Sciences (2nd edition). Hillsdale (NJ): Lawrence Erlbaum Associates, Inc., 1988.

Disclosure of Interests: : Sebastian C Rodriguez-García Speakers bureau: Novartis Farmaceutica, S.A., Merck Sharp \& Dohme España, S.A., Sanof Aventis, UCB Pharma, Raul Castellanos-Moreira Speakers bureau: Lilly, MSD, Sanofi, UCB, Jacqueline Uson Jaeger: None declared, Esperanza Naredo: None declared, Loreto Carmona Grant/research support from: Novartis Farmaceutica SA, Pfizer, S.L.U., Merck Sharp \& Dohme España, S.A., Roche Farma, S.A Sanofi Aventis, AbbVie Spain, S.L.U., and Laboratorios Gebro Pharma, SA (All trhough institution)

DOI: 10.1136/annrheumdis-2020-eular.644 